\title{
From Asteroids to Space Debris
}

\section{Zouhair Benkhaldoun ${ }^{1}$, Hong-Kyu Moon ${ }^{2}$, Ahmed Daassou ${ }^{1}$, Jang-Hyun Park ${ }^{2}$ and Mohamed Lazrek ${ }^{1}$}

\author{
${ }^{1}$ High Energy Physics and Astrophysics Laboratory, Oukaimeden Observatory, Cadi Ayyad \\ University, FSSM, Av Prince My Abdallah, BP2390, Morocco \\ email: zouhair@uca.ma \\ ${ }^{2}$ Korea Astronomy and Space Science Institute, Daejeon, South Korea \\ email: fullmoon@kasi.re.kr
}

\begin{abstract}
Since 2011, Oukaimeden Observatory (OUCA) has become one of the active NEO search facilities in the word. Its discovery statistics shows that the MOSS (Morocco Oukaimeden Sky Survey) project received credits for more than 2, 145 new designations, including 3 NEOs and 4 comets. Its excellent astro-climactic characteristics are partly behind the success. The average number of observable nights is around 280 nights per year, while median seeing is $0.8-0.9$ arcsec. We completed construction of a new telescope at the site in March 2015. It is Optical Wide-field Patrol (OWL) facility designed and built by Korea Space Science Institute (KASI). The primary objective of this facility is to monitor national space assets of Korea; either wide-field imaging- or fast data acquisition- capabilities enable the $0.5 \mathrm{~m}$ telescope to conduct observation programs to catalog and follow-up various transient events in the night sky. We present the seeing condition, the OWL system and preliminary results obtained at OWL@Oukaimeden during the past several months.
\end{abstract}

Keywords. Telescopes, Comets, Minor Planets, Asteroids, Surveys, techniques: image processing.

\section{Introduction}

Oukaimeden Observatory (OUCA) is located on the Moroccan High Atlas Mountains at $2700 \mathrm{~m}$ above sea level $\left(7^{\circ} 52^{\prime} 52^{\prime \prime} \mathrm{W}, 31^{\circ} 12^{\prime} 32^{\prime \prime} \mathrm{N}\right), 70 \mathrm{~km}$ from Marrakesh city. From several site testing measurements on the mountaintop, we obtained median seeing of 0. ." 84 and the mean zenith seeing of $0 . " 92$ at OUCA during the fifteen months of observations (July 2003 - September 2004) in Johnson V-band. The best seeing measured at the site during this period was 0." 32 (Benkhaldoun et al. 2005). The comparaison to the other observatories is presented in Table 1.

Due to its excellent astro-climactic characteristics, several science programs are now being conducted at the Oukaimeden Observatory (Fig. 1).

\section{OWL System}

KASI plans to build a network of five identical Optical Wide-field Patrol (OWL) facilities called OWL-Net. They will be established at locations with well-established reputations for their infrastructures and excellent weather. An OWL station, including OWL@Oukaimeden(Fig. 2), is comprised of a $0.5 \mathrm{~m}$ wide-field telescope, a $4 k$ CCD camera, an all-sky dome, an enclosure, the control units and site utilities. The resulting effective field of view (FOV) of an OWL telescope is 1.75 degrees (diagonal); its plate scale is 0.976 arcsec/pixel. The OWL team achieved high precision tracking and fast slewing rates for acquisition and positioning; the maximum speed for slewing is 10 $\mathrm{deg} / \mathrm{sec}$; the acceleration is $2 \mathrm{deg} / \mathrm{sec}^{2}$. The OWL@Oukaimeden is linked to the internet, 
Table 1. Mean astronomical seeing at some astronomical observatories in the world. For Oukaimeden, we present here the bias-corrected value (Benkhaldoun et al. (2005))

\begin{tabular}{l|c|c|l}
\hline Site & FWHM & Months & References \\
\hline La Palma & 0.76 & 9 & Muñoz-Tuñón et al. 1997 \\
\hline Cerro Paranal & 0.81 & 143 & Sarazin et al. 2003 \\
\hline Mauna Kea & 0.7 & 12 & N.A.O. 2003 \\
\hline La Silla & 0.93 & 158 & Sarazin et al. 1999 \\
\hline Maidanak & 0.76 & 27 & $\mid$ Ehgamberdiev et al. 2000 \\
\hline Oukaïmeden & $\mathbf{1 . 0 5}$ & 15 & Benkhaldoun et al. 2005 \\
\hline
\end{tabular}

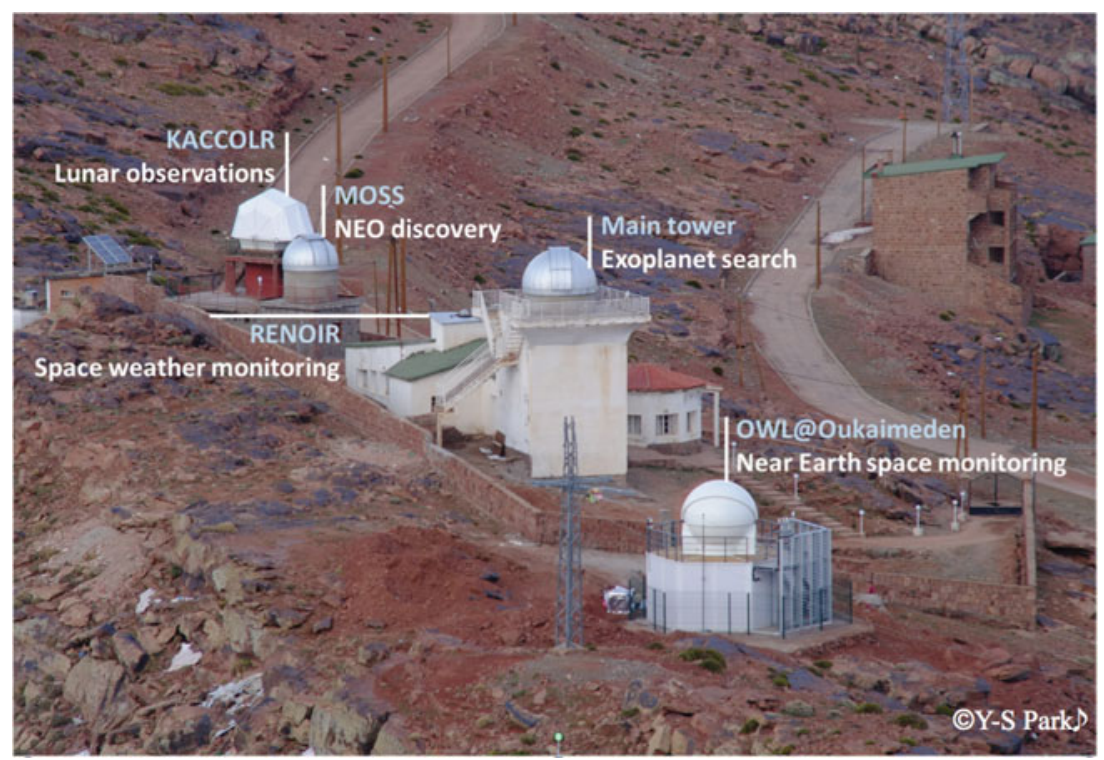

Figure 1. Various domes including OWL@Oukaimeden in OUCA.

and controlled and monitored at KASI in South Korea. The site operations processes of the OWL-Net will be linked, controlled and executed in such a manner that a suite of network operating software maximizes the overall data productivity.

\section{OWL Science}

Due to relatively short duty cycle for space object tracking, OWL facilities are expected to accommodate enough telescope time for science programs. The wide-field capability of the OWL facilities is ideal for detection, follow-up astrometry and photometry of asteroids and comets. An examples of satellite tracklets obtained with the image chopper and the CCD camera during the test observations at OWL@Oukaimeden are presented in (Fig. 3) (Park, J.-H. et al. (2016)). The candidates science projects at OWL@Oukaimeden are:

- Follow-up astrometry of bright Near-earth objects

- Target of opportunity photometry of Solar System small bodies

- Transit observations of extrasolar planets 


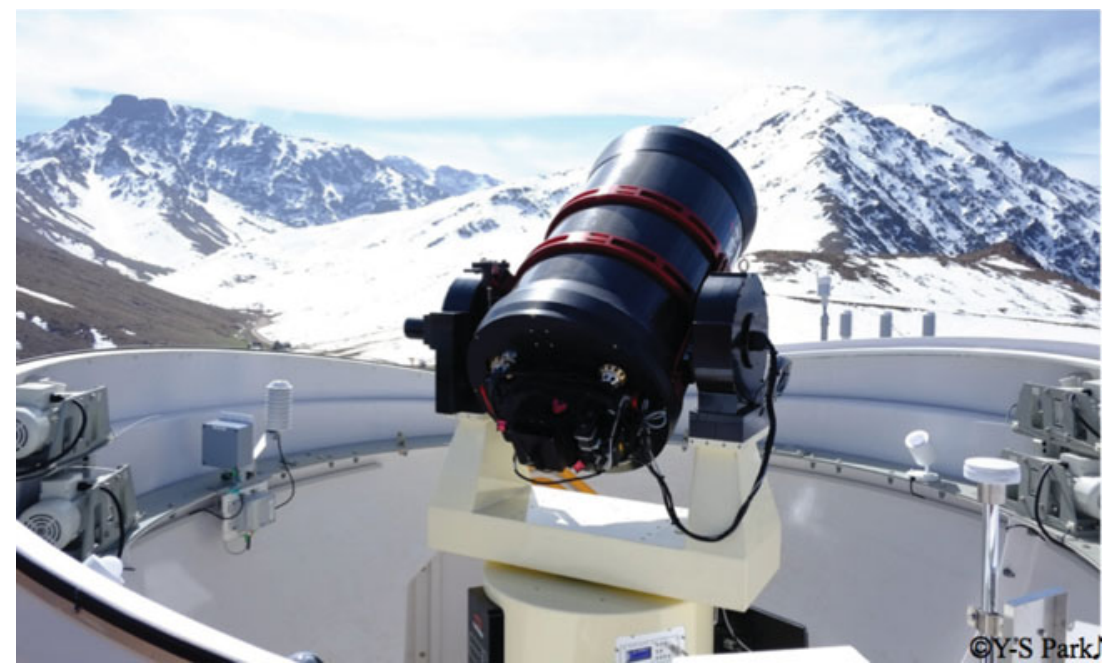

Figure 2. Overview of OWL@Oukaimeden system. (Park, J.-H. et al. (2016))
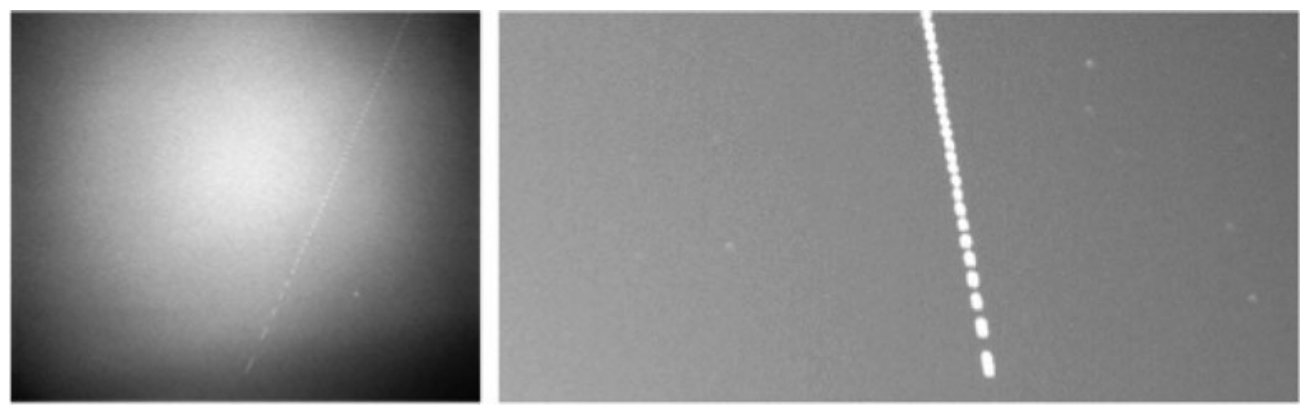

Figure 3. Examples of satellite tracklets obtained with image chopper and CCD camera during test observations at OWL@Oukaimeden. (Park, J.-H. et al. (2016))

\section{Conclusion}

Owl-Net@Oukaimeden has reinforced the already existing infrastructure at the Oukaimeden observatory for the detection and monitoring of Near Earth Objects and comets. Thanks to this newly established facility on the summit, the position of Oukaimeden Observatory has been strengthened as one of the best sites for survey and characterization of bright small Solar System bodies, with its new mission for space debris tracking, despite the fact that it has small-sized telescopes. The preliminary results from the test runs with the OWL@Oukaimeden suggest follow-up fine-tuning of the system.

\section{References}

Benkhaldoun, Z., Abahamid A., El Azhari Y. \& M. Lazrek 2005, A\& A, 30, 490

Park, J.-H. et al. 2016, In preparation 